\title{
Subject Index Vol. 43, 1980
}

Acoustic nerve tumor 189

Algorithm 172

Arteriovenous malformation 164

Basalganglia 133

Biocalibration 244

Biopsy 189,198,216

Blind evaluation 114

Brain mapping 8, 18,28,48,67,183,269

Brain stem 313

Brain tumors 198,164

BsEP 313

'Busy-line' phenomenon 305

\section{Cancer 145}

Cat 269

Caudate injection of alumina cream 37

Center medianum 133

Cerebellar stimulation 114,230

Cerebellum 48,228,230

Cerebral abscess 205

Cerebral palsy 114

Cervical-evok'ed responses 118

Chronic brain stimulation 230

Chronic chemical stimulation 37

CNSlesions 176

Cognitive function 259

Complications 114

Computed tomography 170

Computer 18,28,67

Computerized surgery 176

Computerized tomography 164, 174, 205,

216 Continuous vibration 305 Contrast ventriculography 269 CTreconstruction 210 CTscan 176,183 CT scanning 59,172

Deep brain stimulation $133,244,298$ 
Dentate nucleus 48 DepthRecording 183 Diencephalic topography 18,28,67 3Dimensional reconstruction 176 Dipole 313 DNA 216

Dorsal column stimulation 8,228 Dyskinesia 133,259

Electrical stimulation 89 Electromyography 48 EMG-reaction time 40 Endorphin 150 Epidural recording 118 Epilepsy 230

Evoked potentials 8,48,313 Evoked responses 109

Facilitation 40

Fields of Forel interruption 104

Gate control hypothesis 305 Glioma 164,216

Histopathology 216 Hypophysectomy 145 Hypothermia 1

Inhibition 40 Inhibitionofmotility 37 Internal capsule 244 Interstitial radiotherapy 198 Intracavitary radiation 198 Intracerebral coordinate system 269 Intracerebral hematoma 96 Intracranial neoplasm 210 Intractable epilepsy 104 Intractable pain 128,145 Involuntary movement 150 
Joint units 28

Laser 210

Linear accelerator 164

Lumbosacral-evoked responses 118

Metastatic ca rei no ma 145 Microelectrode recording 263 Microstereoencephalotomy 222

Microsurgery 176 Midbrain 89, 150 Motor control 48 Motor system 109 Movement

disorder 244 'movement units' 67 Multitarget stereotactic surgery 109 Muscle units 28

Muscular hypertonus 298

Neurosurgical localization 170

Pain 8,133,150,228,290,305 Parafascicularis 133 Parkinson surgery 109 Periaqueductal gray matter 150 Phase reversal 313 Phosphenes 89

Positron emission tomography 164 Posterior columns stimulation 228 Posture 40

Pressure units 18 Pulvinar 128 Pulvinotomy 128 
Rigidity 263

Shelden tumorscope 176

Somatosensory evoked potential 1

Somatosensory evoked responses 244

Spasmodic torticollis 159

Spasticity 228,230

Stereotactic evacuation 96

Stereotactic diagnosis 198

Stereotactic surgery $8,18,28,59,67,128,133,145,159,170,172,174,176,183,189$,

$205,210,216,222,244,259,263$

Stereotactic surgery for epilepsy 104

Stereotactic techniques 164,210

Stereotaxis 48

Stimulation 133 .

Stimulation-produced analgesia 290

Striatum 37

Sural nerve action potential 305

Surface recording 118

Thalamic cellular activity $18,28,67$ Thalamotomy 8,159,259 Thalamus 89,133,222,

244,259,263 Therapeuticbrain stimulation 298 Tonic vibration reflex 298 Transcutaneous stimulation 290 Tremor 222,263

Ventriculography 59 Visual Effects 89

Water-soluble contrast media 59

Rageattacks 59 


\section{Zonaincerta 150}

